DESY 01-024

hep-ph/0103185

\title{
Pair Production from Vacuum at the Focus of an X-Ray Free Electron Laser
}

\author{
A. Ringwald \\ Deutsches Elektronen-Synchrotron DESY, Hamburg, Germany
}

\begin{abstract}
There are definite plans for the construction of X-ray free electron lasers (FEL), both at DESY, where the so-called XFEL is part of the design of the electron-positron linear collider TESLA, as well as at SLAC, where the so-called Linac Coherent Light Source (LCLS) has been proposed. Such an X-ray laser would allow for high-field science applications: One could make use of not only the high energy and transverse coherence of the X-ray beam, but also of the possibility of focusing it to a spot with a small radius, hopefully in the range of the laser wavelength. Along this route one obtains very large electric fields, much larger than those obtainable with any optical laser of the same power. In this letter we discuss the possibility of obtaining an electric field so high that electron-positron pairs are spontaneously produced in vacuum (Schwinger pair production). We find that if X-ray optics can be improved to approach the diffraction limit of focusing, and if the power of the planned X-ray FELs can be increased to the terawatt region, then there is ample room for an investigation of the Schwinger pair production mechanism.
\end{abstract}


1. Spontaneous particle creation from vacuum induced by an external field, first put forth to examine the production of electron-positron $\left(e^{+} e^{-}\right)$pairs in a static, spatially uniform electric field [1, 2, 3] and often referred to as the Schwinger mechanism, ranks among the most intriguing nonlinear phenomena in quantum field theory. Its consideration is theoretically important, since it requires one to go beyond perturbation theory, and its experimental observation would verify the validity of the theory in the domain of strong fields. Moreover, this mechanism has been applied to many problems in contemporary physics, ranging from black hole quantum evaporation [4, 5, 6, 7. to particle production in hadronic collisions [8, 9, 10, and in the early universe [11, 12, to mention only a few. One may consult the monographs [13, 14, 15] for a review of further applications, concrete calculations and a detailed bibliography.

It is known since a long time that in the background of a static, spatially uniform electric field the vacuum in quantum electrodynamics (QED) is unstable and, in principle, sparks with spontaneous emission of $e^{+} e^{-}$pairs [1, 2, 3]. However, a sizeable rate for spontaneous pair production requires extraordinary strong electric field strengths $\mathcal{E}$ of order or above the critical value

$$
\mathcal{E}_{c} \equiv \frac{m_{e} c^{2}}{e \lambda_{e}}=\frac{m_{e}^{2} c^{3}}{e \hbar} \simeq 1.3 \times 10^{18} \mathrm{~V} / \mathrm{m}
$$

Otherwise, for $\mathcal{E} \ll \mathcal{E}_{c}$, the work of the field on a unit charge $e$ over the Compton wavelength of the electron $\lambda_{e}=\hbar /\left(m_{e} c\right)$ is much smaller than the rest energy $2 m_{e} c^{2}$ of the produced $e^{+} e^{-}$ pair, the process can occur only via quantum tunneling, and its rate is exponentially suppressed, $\propto \exp \left(-\pi \mathcal{E}_{c} / \mathcal{E}\right)$.

Unfortunately, it seems inconceivable to produce macroscopic static fields with electric field strengths of the order of the Schwinger critical field (11) in the laboratory. In view of this difficulty, in the early 1970's the question was raised 1 whether intense optical lasers could be employed to study the Schwinger mechanism [19, 20]. Yet, it was found that all available and conceivable optical lasers did not have enough power density to allow for a sizeable pair creation rate [19, 20, 21, 22, 23, 24, 25, 26, 27, 28, 29, 30].

Meanwhile, there are definite plans for the construction of X-ray free electron lasers (FEL), both at DESY, where the so-called XFEL is part of the design of the $e^{+} e^{-}$linear collider TESLA 31, 32, 33, as well as at SLAC, where the so-called Linac Coherent Light Source (LCLS) has been proposed [34, 35. It has been pointed out by several authors 36, 37, 38, 39] that an X-ray laser would allow for high-field science applications: One could make use of not only the high energy and transverse coherence of the X-ray beam, but also of the possibility of focusing it to a spot with a small radius $\sigma$, hopefully in the range of the laser wavelength, $\sigma \gtrsim \lambda \simeq \mathcal{O}(0.1) \mathrm{nm}$. In this way one might obtain very large electric fields, $\mathcal{E} \propto 1 / \sigma \sim 1 / \lambda$, much larger than those obtainable with any optical laser of the same power.

Electron-positron pair production at the focus of an X-ray FEL has been discussed in Ref. [36 2 and an estimate of the corresponding rate has been presented in Ref. [37]. It is the purpose of

\footnotetext{
${ }^{1}$ At about the same time, the thorough investigation of the question started whether the necessary superstrong fields around $\mathcal{E}_{c}$ can be generated microscopically and transiently in the Coulomb field of colliding heavy ions with $Z_{1}+Z_{2}>Z_{c} \approx 170$ [16, 17]. At the present time, clear experimental signals for spontaneous positron creation in heavy ion collisions are still missing and could only be expected from collisions with a prolonged lifetime (for a recent status report of this issue, see Ref. [18]).

${ }^{2}$ In Ref. 36] the production of positrons in the collision of $46.6 \mathrm{GeV} / \mathrm{c}$ electrons with terawatt optical laser
} 
this letter to strengthen these considerations and to present a state of the art evaluation of the prospects to observe the Schwinger mechanism at future X-ray laser facilities. In particular, we determine critical laser parameters, like the laser power and the focus spot size, which should be aimed at to get an observable effect. To this end, we make heavily use of the rather well forgotten work on the Schwinger mechanism in alternating electric fields by Russian groups [21, 22, 24, 25, 26, 27, 28, 29, 30].

2. We start with a discussion of a number of simplifying approximations concerning the electromagnetic field of the laser radiation. We elaborate on a model which retains the main features of the general case but nevertheless allows to obtain final expressions for the pair production rate in closed form. This should be sufficient for an order-of-magnitude estimate of the critical parameters.

It is well known that no pairs are produced in the background of a light-like static, spatially uniform electromagnetic field [3], characterized invariantly by

$$
\begin{aligned}
\mathcal{F} & \equiv \frac{1}{4} F_{\mu \nu} F^{\mu \nu} \equiv-\frac{1}{2}\left(\mathbf{E}^{2}-c^{2} \mathbf{B}^{2}\right)=0 \\
\mathcal{G} & \equiv \frac{1}{4} F_{\mu \nu} \tilde{F}^{\mu \nu} \equiv c \mathbf{E} \cdot \mathbf{B}=0
\end{aligned}
$$

where $F^{\mu \nu}$ is the electromagnetic field strength tensor and $\tilde{F}^{\mu \nu}=(1 / 2) \epsilon^{\mu \nu \alpha \beta} F_{\alpha \beta}$ its dual. It has been argued that fields produced in (optical) focusing of laser beams are very close to such a light-like electromagnetic field, leading to an essential suppression of pair creation 4 [23]. For other fields, $\mathcal{F}$ and $\mathcal{G}$ do not vanish, and pair production becomes possible, unless $\mathcal{G}=0, \mathcal{F}>0$, corresponding to a pure magnetic field in an appropriate coordinate system [3. In particular, one expects pair creation in the background of a spatially uniform electric field oscillating with a frequency $\omega$, say

$$
\mathbf{E}(t)=(0,0, \mathcal{E} \cos (\omega t)), \quad \mathbf{B}(t)=(0,0,0),
$$

which has $\mathcal{G}=0, \mathcal{F}<0$. As emphasized in Refs. [24, 25, 28, 30, 37], such a field may be created in an antinode of the standing wave produced by a superposition of two coherent laser beams with wavelength

$$
\lambda=\frac{2 \pi c}{\omega}
$$

and, indeed, it may be considered as spatially uniform at distances much less than the wavelength. Thus, for definiteness, we assume that every X-ray laser pulse is split into two equal parts and recombined to form a standing wave with locations where the electromagnetic field has the form (4) and where the peak electric field is given by $\left(1 \mathrm{TW}=10^{12} \mathrm{~W}\right)$

$$
\mathcal{E}=\sqrt{\mu_{0} c \frac{P}{\pi \sigma^{2}}} \simeq 1.1 \times 10^{17}\left(\frac{P}{1 \mathrm{TW}}\right)^{1 / 2}\left(\frac{0.1 \mathrm{~nm}}{\sigma}\right) \frac{\mathrm{V}}{\mathrm{m}},
$$

pulses, observed by the SLAC experiment E-144 [40, was discussed. Whereas in Ref. 40] the data were interpreted in terms of multiphoton light-by-light scattering, an alternative explanation in terms of the Schwinger mechanism was offered in Ref. [36] (see also Ref. [41]).

${ }^{3}$ Unless otherwise stated, we use the rationalized MKSA unit system throughout. For the numerical values of the physical constants we take the ones given in Ref. [42].

${ }^{4}$ Yet, in a focused wave there are regions near the focus where $\mathcal{F}<0$ and pair production is possible [19, 36]. 


\begin{tabular}{|c|c|c|c|c|c|}
\hline \multicolumn{6}{|c|}{ Laser Parameters } \\
\hline & & \begin{tabular}{l|l|} 
Optical 43 \\
\end{tabular} & \multicolumn{3}{|c|}{$\overline{\text { X-ray FEL }}$} \\
\hline & & $\begin{array}{c}\text { Focus: } \\
\text { Diffraction limit }\end{array}$ & Design 33 & $\begin{array}{c}\text { Focus: } \\
\text { Available } 44\end{array}$ & $\begin{array}{c}\text { Focus: } \\
\text { Goal } 37\end{array}$ \\
\hline Wavelength & $\bar{\lambda}$ & $1 \mu \mathrm{m}$ & $0.4 \mathrm{~nm}$ & $0.4 \mathrm{~nm}$ & $0.15 \mathrm{~nm}$ \\
\hline Photon energy & $\hbar \omega=\frac{h c}{\lambda}$ & $1.2 \mathrm{eV}$ & $3.1 \mathrm{keV}$ & $3.1 \mathrm{keV}$ & $8.3 \mathrm{keV}$ \\
\hline Peak power & $P \quad{ }^{\lambda}$ & $1 \mathrm{PW}$ & $110 \mathrm{GW}$ & $1.1 \mathrm{GW}$ & $5 \mathrm{TW}$ \\
\hline Spot radius (rms) & $\sigma$ & $1 \mu \mathrm{m}$ & $26 \mu \mathrm{m}$ & $21 \mathrm{~nm}$ & $0.15 \mathrm{~nm}$ \\
\hline Coherent spike length (rms) & $\triangle t$ & 500 fs $\div 20 \mathrm{ps}$ & $0.04 \mathrm{fs}$ & $0.04 \mathrm{fs}$ & $0.08 \mathrm{ps}$ \\
\hline \multicolumn{6}{|c|}{ Derived Quantities } \\
\hline Peak power density & $S=\frac{P}{\pi \sigma^{2}}$ & $3 \times 10^{26} \frac{\mathrm{W}}{\mathrm{m}^{2}}$ & $5 \times 10^{19} \frac{\mathrm{W}}{\mathrm{m}^{2}}$ & $8 \times 10^{23} \frac{\mathrm{W}}{\mathrm{m}^{2}}$ & $7 \times 10^{31} \frac{\mathrm{W}}{\mathrm{m}^{2}}$ \\
\hline Peak electric field & $\mathcal{E}=\sqrt{\mu_{0} c S}$ & $4 \times 10^{14} \frac{\mathrm{v}}{\mathrm{m}}$ & $1 \times 10^{11} \frac{\mathrm{v}}{\mathrm{m}}$ & $2 \times 10^{13} \frac{\mathrm{v}}{\mathrm{m}}$ & $2 \times 10^{17} \frac{\mathrm{v}}{\mathrm{m}}$ \\
\hline Peak electric field/critical field & $\mathcal{E} / \mathcal{E}_{c}$ & $3 \times 10^{-4}$ & $1 \times 10^{-7}$ & $1 \times 10^{-5}$ & 0.1 \\
\hline Photon energy/e rest energy & $\frac{\hbar \omega}{m_{e} c^{2}}$ & $2 \times 10^{-6}$ & 0.006 & 0.006 & 0.02 \\
\hline Adiabaticity parameter & $\gamma=\frac{\hbar \omega}{e \mathcal{E} \lambda_{e}}$ & $9 \times 10^{-3}$ & $6 \times 10^{4}$ & $5 \times 10^{2}$ & 0.1 \\
\hline
\end{tabular}

Table 1: Laser parameters and derived quantities relevant for estimates of the rate of spontaneous $e^{+} e^{-}$pair production. The column labeled "Optical" lists parameters which are typical for a petawatt-class $\left(1 \mathrm{PW}=10^{15} \mathrm{~W}\right)$ optical laser [43], focused to the diffraction limit, $\sigma=\lambda$. The column labeled "Design" displays design parameters of the planned XFEL at DESY ("SASE-5" in Ref. [33]). Similar values apply for LCLS [34, 35]. The column labeled "Focus: Available" shows typical values which can be achieved with present day methods of X-ray focusing [44, 45]: It assumes that the XFEL X-ray beam can be focused to a rms spot radius of $\sigma \simeq 21 \mathrm{~nm}$ with an energy extraction efficiency of $1 \%$ [44. The column labeled "Focus: Goal" shows parameters which are theoretically possible by increasing the energy extraction of LCLS (by the tapered undulator technique) and by a yet unspecified method of diffraction-limited focusing of X-rays 37 .

in terms of the laser power $P$, the focus spot radius $\sigma$ and the permeability of free space $\mu_{0}=$ $4 \pi \times 10^{-7} \mathrm{~N} \mathrm{~A}^{-2}$. Furthermore, we assume that the peak electric field $\mathcal{E}$ is much smaller than the Schwinger critical field (11) and the energy of the laser photons is much smaller than the rest energy of the electron,

$$
\mathcal{E} \ll \mathcal{E}_{c}=\frac{m_{e}^{2} c^{3}}{e \hbar}, \quad \quad \hbar \omega \ll m_{e} c^{2} ;
$$

conditions which are well satisfied at realistic optical as well as X-ray lasers (c. f. Table 1).

3. Under these conditions, it is possible to compute the rate of $e^{+} e^{-}$pair production in a semiclassical manner, using generalized WKB [20] or imaginary-time [21, 22, 27, 29] methods (see also Ref. [46] and references cited therein). Let us summarize the basic results of the corresponding studies.

In Ref. [20, the probability that an $e^{+} e^{-}$pair is produced per unit time and unit volume,

$$
w=\frac{\mathrm{d} n_{e^{+} e^{-}}}{\mathrm{d}^{3} x \mathrm{~d} t},
$$


was estimated as

$$
w_{\mathrm{BI}}=\frac{c}{4 \pi^{3} \lambda_{e}{ }^{4}}\left(\frac{\mathcal{E}}{\mathcal{E}_{c}}\right)^{2} \frac{\pi}{g(\gamma)+\frac{1}{2} \gamma g^{\prime}(\gamma)} \exp \left[-\pi \frac{\mathcal{E}_{c}}{\mathcal{E}} g(\gamma)\right]
$$

with [20, 21]

$$
\begin{aligned}
g(\gamma) \equiv \frac{4}{\pi} \int_{0}^{1} \mathrm{~d} u\left[\frac{1-u^{2}}{1+\gamma^{2} u^{2}}\right]^{1 / 2} & =\frac{4}{\pi} \frac{\sqrt{1+\gamma^{2}}}{\gamma^{2}}\left[\boldsymbol{K}\left(\frac{\gamma}{\sqrt{1+\gamma^{2}}}\right)-\boldsymbol{E}\left(\frac{\gamma}{\sqrt{1+\gamma^{2}}}\right)\right] \\
& = \begin{cases}1-\frac{1}{8} \gamma^{2}+\mathcal{O}\left(\gamma^{4}\right) \quad: \gamma \ll 1, \\
\frac{4}{\pi \gamma} \ln \left(\frac{4 \gamma}{\mathrm{e}}\right)+\mathcal{O}\left(1 / \gamma^{3}\right): & \gamma \gg 1 .\end{cases}
\end{aligned}
$$

Here, $\boldsymbol{K}$ and $\boldsymbol{E}$ are the complete elliptic integrals of the first and second kind, respectively.

The laser frequency $\omega$ enters in the semiclassical formula (99) only through the ratio $\gamma$ of the energy of the laser photons over the work of the field on a unit charge $e$ over the Compton wavelength of the electron,

$$
\gamma \equiv \frac{\hbar \omega}{e \mathcal{E} \lambda_{e}}=\frac{\hbar \omega}{m_{e} c^{2}} \frac{\mathcal{E}_{c}}{\mathcal{E}}=\frac{m_{e} c \omega}{e \mathcal{E}} \equiv \frac{\mathcal{E}_{\omega}}{\mathcal{E}}
$$

which can take on arbitrary values despite of the conditions (7). This ratio plays the rôle of an adiabaticity parameter, as is easily inferred from the asymptotic behaviour (11) of the function $g(\gamma)$, which enters in the principal, exponential factor in the pair production probability (9). Indeed, as long as $\gamma \ll 1$, i. e. in the high-field, low-frequency limit, formula (9) agrees with the nonperturbative result from Ref. [3] for a static, spatially uniform field,

$$
w_{\mathrm{S}}=\frac{c}{4 \pi^{3} \lambda_{e}{ }^{4}}\left(\frac{\mathcal{E}}{\mathcal{E}_{c}}\right)^{2} \sum_{\ell=1}^{\infty} \frac{1}{\ell^{2}} \exp \left[-\ell \pi \frac{\mathcal{E}_{c}}{\mathcal{E}}\right] \stackrel{\mathcal{E} \lll \mathcal{E}_{c}}{\cong} \frac{c}{4 \pi^{3} \lambda_{e}^{4}}\left(\frac{\mathcal{E}}{\mathcal{E}_{c}}\right)^{2} \exp \left[-\pi \frac{\mathcal{E}_{c}}{\mathcal{E}}\right]
$$

apart from an "inessential" (c.f. Ref. [20]) pre-exponential factor of $\pi$. In this case, $e^{+} e^{-}$pair production at a laser has all the features of the usual tunneling effect. On the other hand, for $\gamma \gg 1$, i. e. in the low-field, high-frequency limit, formula (9) ressembles a perturbative result,

$$
w_{\mathrm{BI}} \simeq \frac{c}{4 \pi^{3} \lambda_{e}{ }^{4}}\left(\frac{\hbar \omega}{m_{e} c^{2}}\right)^{2} \frac{\pi}{2 \gamma \ln (4 \gamma)}\left(\frac{\mathrm{e}}{4 \gamma}\right)^{2 \frac{2 m_{e} c^{2}}{\hbar \omega}}\left(1+\mathcal{O}\left(\frac{1}{\gamma^{2}}\right)\right), \quad \text { for } \gamma \gg 1
$$

Since $\gamma \propto 1 / e$, formula (14) corresponds to the $n$-th order perturbation theory, $n$ being the minimum number of quanta required to create an $e^{+} e^{-}$pair: $n \gtrsim 2 m_{e} c^{2} /(\hbar \omega) \gg 1$. Therefore, expression (9) for the pair production rate interpolates analytically between the adiabatic, nonperturbative tunneling mechanism $(\gamma \ll 1)$ and the anti-adiabatic, perturbative multi-photon production mechnism $(\gamma \gg 1)$.

The principal, exponential factor, $\exp \left[-\pi\left(\mathcal{E}_{c} / \mathcal{E}\right) g(\gamma)\right]$, in the pair production probability (91) has been confirmed by later work [21, 22, 27, 29]. However, the imaginary-time method, exploited by these later studies, allowed to determine the pre-exponential factor more accurately, by taking 
into account also interference effects. It was found [27, 29] that the pair production probability $w$, under the conditions (17), can be represented as a sum of probabilities $w_{n}$ of many-photon processes,

$$
w_{\mathrm{P}}=\sum_{n>n_{0}} w_{n}, \quad \text { with } n_{0}=\frac{m_{e} c^{2}}{\hbar \omega} \triangle
$$

where the latter are given 5 in terms of an integral over the three momentum components $\mathbf{p}=$ $\left(\mathbf{p}_{\perp}, p_{\|}\right)$, of the produced $e^{-}$(or, equivalently, $\left.e^{+}\right)$, perpendicular $\left(\mathbf{p}_{\perp}\right)$ and parallel $\left(p_{\|}\right)$to the applied electric field (4), respectively,

$$
\begin{aligned}
w_{n}= & \frac{2}{\pi} \omega^{2} \exp \left[-\pi \frac{\mathcal{E}_{c}}{\mathcal{E}} g(\gamma)\right] \int \frac{\mathrm{d}^{3} p}{(2 \pi)^{3}}\left[1-(-)^{n} \cos \left(4 \frac{p_{\|}}{\omega} \arctan \gamma\right)\right] \times \\
& \times \exp \left[-\pi \frac{\mathcal{E}_{c}}{\mathcal{E}}\left\{\left(g(\gamma)+\frac{1}{2} \gamma g^{\prime}(\gamma)\right) \frac{\mathbf{p}_{\perp}^{2}}{m_{e}^{2}}-\gamma \frac{\mathrm{d}}{\mathrm{d} \gamma}\left(g(\gamma)+\frac{1}{2} \gamma g^{\prime}(\gamma)\right) \frac{p_{\|}^{2}}{m_{e}^{2}}\right\}\right] \times \\
& \times \delta\left(\triangle(\gamma)+\frac{1}{2}\left(1+\gamma \frac{\mathrm{d}}{\mathrm{d} \gamma}\right) \triangle(\gamma) \frac{\mathbf{p}_{\perp}^{2}}{m_{e}^{2}}+\frac{1}{2}\left(1-\gamma \frac{\mathrm{d}}{\mathrm{d} \gamma}\right)\left(1+\gamma \frac{\mathrm{d}}{\mathrm{d} \gamma}\right) \triangle(\gamma) \frac{p_{\|}^{2}}{m_{e}^{2}}-n \frac{\omega}{m_{e}}\right) .
\end{aligned}
$$

In Eqs. (15) and (16), the function $\triangle(\gamma)$,

$$
\triangle(\gamma)=\frac{4}{\pi} \frac{\sqrt{1+\gamma^{2}}}{\gamma} \boldsymbol{E}\left(\frac{1}{\sqrt{1+\gamma^{2}}}\right)= \begin{cases}\frac{4}{\pi \gamma}\left(1-\frac{1}{2} \gamma^{2} \ln \left(\frac{\gamma}{4 \sqrt{\mathrm{e}}}\right)+\mathcal{O}\left(\gamma^{4}\right)\right) & : \gamma \ll 1 \\ 2+\frac{1}{2} \frac{1}{\gamma^{2}}+\mathcal{O}\left(1 / \gamma^{4}\right) & : \gamma \gg 1\end{cases}
$$

plays the rôle of an effective gap width between the lower and upper continuum (in units of $\left.m_{e} c^{2}\right)$. The presence of the factor $\cos \left(4\left(p_{\|} / \omega\right) \arctan \gamma\right)$ in the rate (16) results in oscillations 6 in the momentum spectrum, mostly manifest at $p_{\|}=0$ (see also Refs. [26, 28]). In this case $w_{n} \propto 1-(-)^{n}$, i. e. electrons are created by odd harmonics. This selection rule is due to interference and related to particle statistics. The argument of the of the delta function in formula (15) expresses conservation of energy and determines the dispersion law for the electron in the strong external field.

The integration over the three-momentum $\mathbf{p}$ in formula (16) can be performed analytically and the result can be expressed in terms of special functions [29]. For our purpose, however, we need only the limiting cases of small and large $\gamma$, which are even simpler,

$$
\begin{aligned}
w_{\mathrm{P}} \simeq & \frac{c}{4 \pi^{3} \lambda_{e}{ }^{4}} \times \\
& \times \begin{cases}\frac{\sqrt{2}}{\pi}\left(\frac{\mathcal{E}}{\mathcal{E}_{c}}\right)^{\frac{5}{2}} \exp \left[-\pi \frac{\mathcal{E}_{c}}{\mathcal{E}}\left(1-\frac{1}{8} \gamma^{2}+\mathcal{O}\left(\gamma^{4}\right)\right)\right], & : \gamma \ll 1, \\
\sqrt{\frac{\pi}{2}}\left(\frac{\hbar \omega}{m_{e} c^{2}}\right)^{\frac{5}{2}} \sum_{n>2 \frac{m_{e} c^{2}}{\hbar \omega}}\left(\frac{\mathrm{e}}{4 \gamma}\right)^{2 n} \mathrm{e}^{-2\left(n-2 \frac{m_{e} c^{2}}{\hbar \omega}\right)} \operatorname{Erfi}\left(\sqrt{2\left(n-2 \frac{m_{e} c^{2}}{\hbar \omega}\right)}\right) & : \gamma \gg 1,\end{cases}
\end{aligned}
$$

where Erfi is the imaginary error function [48. Both asymptotics bear a neat similarity to the corresponding asymptotics of formula (91). Moreover, the result (18) is, in the adiabatic limit

\footnotetext{
${ }^{5}$ For notationally simplicity, we use $\hbar=c=1$ in Eq. (16).

${ }^{6}$ Analogous oscillations play an important rôle in the description of (p)reheating after inflation [4].
} 
$(\gamma \ll 1)$, in agreement with the classical result (13) of Schwinger, if one properly averages the latter over an oscillation period.

The conditions (17) give an indication on the range of validity of the quoted results. In Refs. [27, 29, 30, it has been argued that for $\mathcal{E} \gtrsim 0.1 \mathcal{E}_{c}$ the backreaction of the produced $e^{+} e^{-}$pairs on the external field and the mutual interactions between these particles has to be taken into account These effects in the superstrong field regime, which are expected to lead to the formation of a plasma, can in principle be accounted for with the help of methods developed in Refs. 49, 50, 51, 52, 53, 54, 55, 56, 57, mainly in the context of particle production in the central rapidity region in heavy ion collisions. The discussion of the plasma regime is however beyond the scope of the present letter, in which we just want to estimate the onset of the Schwinger mechanism.

4. Let us apply now the theoretical rate estimates reviewed above in order to determine the critical laser parameters which should be aimed at to get an observable effect.

In Fig. 1 we display the probability density $w$ as a function of the peak electric field $\mathcal{E}$, for laser photon energies $\hbar \omega \leq 10 \mathrm{keV}$, as applicable at presently planned lasers (c. f. Table 11). In order to appreciate the scale of $w$ in Fig. 1, we note that the Compton space-time volume of an electron has the size

$$
\triangle V_{e} \times \triangle T_{e}=\lambda_{e}{ }^{3} \times\left(\lambda_{e} / c\right) \simeq 7.4 \times 10^{-59} \mathrm{~m}^{3} \mathrm{~s} .
$$

In other words, the probability that an $e^{+} e^{-}$pair is produced within one Compton space-time volume is very small in Fig. 1] $w \triangle V_{e} \triangle T_{e} \ll 1$, as it should be under the conditions (77). Furthermore, we observe that formula (9) overestimates the rate in the considered range of $\mathcal{E}$ by about one order of magnitude. Last but not least we note that in the considered range of the electric field, $\mathcal{E} \gtrsim 10^{17} \mathrm{~V} / \mathrm{m}$, the dependence on the laser frequency $\omega$ is very weak and nearly invisible. This is due to the fact that the adiabatic, nonperturbative, strong field regime, $\gamma \lesssim 1$, starts to apply for $\mathcal{E} \gtrsim \mathcal{E}_{\omega} \equiv \hbar \omega \mathcal{E}_{c} /\left(m_{e} c^{2}\right) \sim 10{ }^{15 \div 16} \mathrm{~V} / \mathrm{m}$ (c.f. Eq. (12) ), for $\hbar \omega \sim 1 \div 10 \mathrm{keV}$.

Let $\triangle V=\sigma^{3}$ be the effective volume, where the peak electric field (6) exists, and consider the average number of $e^{+} e^{-}$pairs per unit time, produced in this volume,

$$
\frac{\mathrm{d} n_{e^{+} e^{-}}}{d t}=w \triangle V
$$

In Fig. 2 we plot this quantity as a function of the laser power $P$ for different laser spot radii $\sigma$, under the assumption that the diffraction limit of focusing, $\sigma=\lambda$ and $\triangle V=\lambda^{3}$, can be reached. From this figure and the typical duration times $\Delta t \sim 10^{-(13 \div 16)} \mathrm{s}$ of the coherent laser pulses presently discussed (c.f. Table 1), we infer that a minimum power of $2.5 \div 4.5 \mathrm{TW}$, corresponding to a electric field of $(1.7 \div 2.3) \times 10^{17} \mathrm{~V} / \mathrm{m}$, is needed to produce at least one $e^{+} e^{-}$ pair, $n_{e^{+} e^{-}}=w \triangle V \triangle t \sim 1$, if the laser has a wavelength of $0.1 \mathrm{~nm}$ and the theoretical diffraction limit is actually reached. For larger wavelength or in the case that the diffraction limit cannot be reached $(\sigma \gg \lambda)$, as is presently the case with existing technology 44, 45] (c. f. column labeled "Focus: Available" in Table 1), this critical power increases considerably. For example, for a spot radius $\sigma=20 \mathrm{~nm}$, the critical power rises to $38 \div 55 \mathrm{PW}$, corresponding to a electric field of $(1.1 \div 1.3) \times 10^{17} \mathrm{~V} / \mathrm{m}$.

The minimally required power $P_{\text {min }}$, the corresponding power density $S_{\text {min }}$ and electric field $\mathcal{E}_{\text {min }}$, to produce at least one $e^{+} e^{-}$pair in a volume $\triangle V=\sigma^{3}$ during a time interval $\triangle t$ at the focus of 


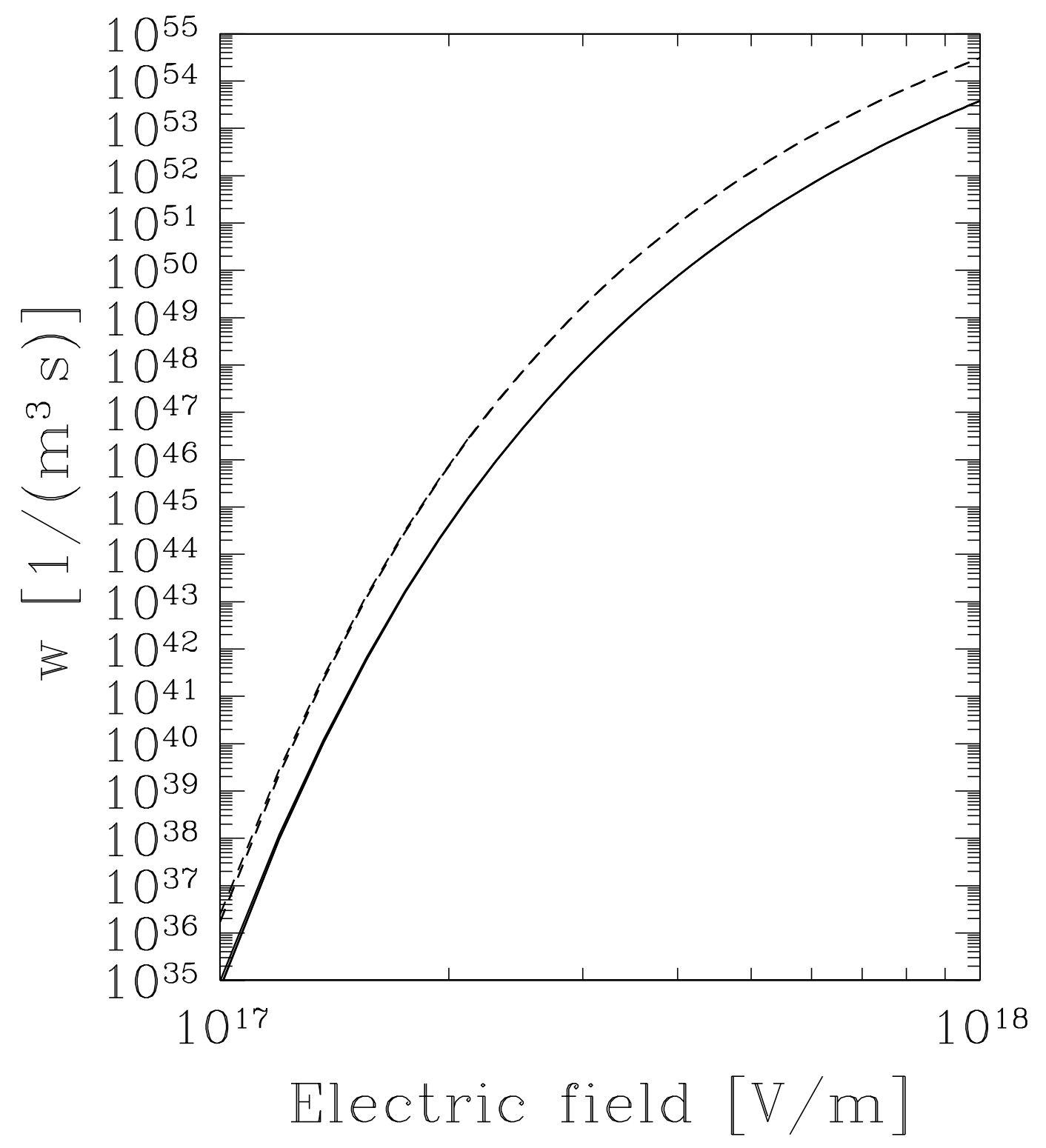

Figure 1: The probability $w$ to produce an $e^{+} e^{-}$pair per unit volume and unit time at a laser, with $E_{\gamma}=\hbar \omega \leq 10 \mathrm{keV}$, as a function of the peak electric field $\mathcal{E}$. The solid and dashed lines refer to the estimates (15) and (9), respectively. The dependence on the laser frequency $\omega$ is very weak and only visible at the lower end of the considered range of the electric field. The respective lower curves correspond to $E_{\gamma}=1 \mathrm{keV}$, the upper ones to $E_{\gamma}=10 \mathrm{keV}$. We note, that the probability that an $e^{+} e^{-}$pair is produced within one Compton space-time volume is very small, $w \cdot \lambda_{e}{ }^{4} / c \approx w \cdot 10^{-58} \mathrm{~m}^{3} \mathrm{~s} \ll 1$, in the whole range of $\mathcal{E}$ considered. 


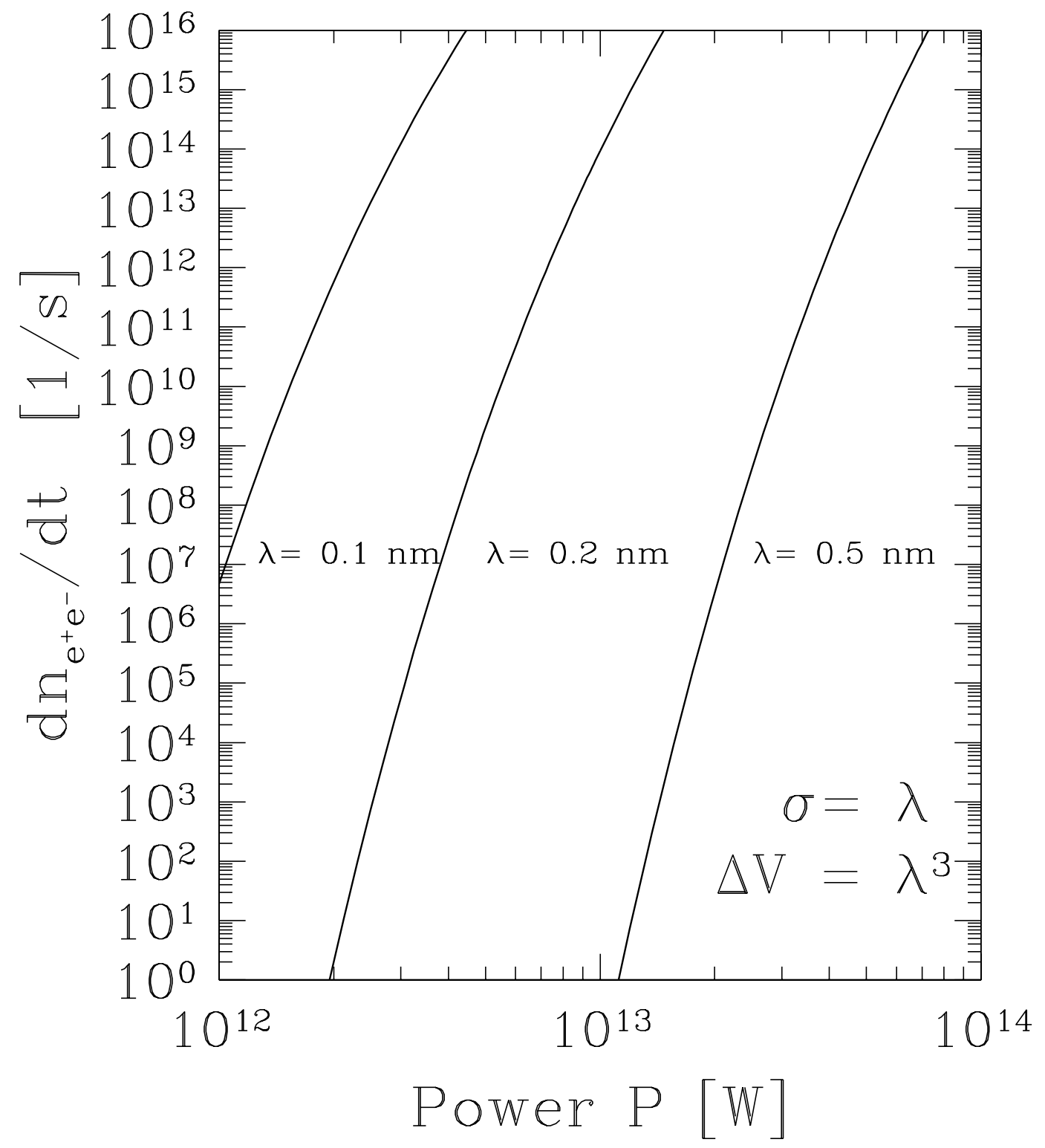

Figure 2: The average number (201) of $e^{+} e^{-}$pairs produced per unit time, $w \triangle V$, where formula (15) is taken for the pair production probability $w$, as a function of the laser power $P$ for different values of the laser wavelength $\lambda$. Here it is assumed that the diffraction limit, $\sigma=\lambda$ and $\triangle V=\lambda^{3}$, can be reached. 


\begin{tabular}{|l|c|c|c||c|c|c|}
\hline & $\lambda$ & $\sigma$ & $\triangle t$ & $P_{\min }$ & $S_{\min }$ & $\mathcal{E}_{\min }$ \\
\hline \hline Focused X-ray FEL: & $0.1 \mathrm{~nm}$ & $0.1 \mathrm{~nm}$ & $0.1 \mathrm{ps}$ & $2.5 \mathrm{TW}$ & $7.8 \times 10^{31} \mathrm{~W} / \mathrm{m}^{2}$ & $1.7 \times 10^{17} \mathrm{~V} / \mathrm{m}$ \\
$(\approx$ "Goal" in Table $1 \mathrm{I})$ & $0.1 \mathrm{~nm}$ & $0.1 \mathrm{~nm}$ & $0.1 \mathrm{fs}$ & $4.5 \mathrm{TW}$ & $1.4 \times 10^{32} \mathrm{~W} / \mathrm{m}^{2}$ & $2.3 \times 10^{17} \mathrm{~V} / \mathrm{m}$ \\
\hline Focused X-ray FEL: & $0.1 \mathrm{~nm}$ & $20 \mathrm{~nm}$ & $0.1 \mathrm{ps}$ & $38 \mathrm{PW}$ & $3.0 \times 10^{31} \mathrm{~W} / \mathrm{m}^{2}$ & $1.1 \times 10^{17} \mathrm{~V} / \mathrm{m}$ \\
$(\approx$ "Available" in Table $1 \mathrm{I})$ & $0.1 \mathrm{~nm}$ & $20 \mathrm{~nm}$ & $0.1 \mathrm{fs}$ & $55 \mathrm{PW}$ & $4.3 \times 10^{31} \mathrm{~W} / \mathrm{m}^{2}$ & $1.3 \times 10^{17} \mathrm{~V} / \mathrm{m}$ \\
\hline \hline Focused Optical Laser: & $1 \mu \mathrm{m}$ & $1 \mu \mathrm{m}$ & $10 \mathrm{ps}$ & $49 \mathrm{EW}$ & $1.6 \times 10^{31} \mathrm{~W} / \mathrm{m}^{2}$ & $7.7 \times 10^{16} \mathrm{~V} / \mathrm{m}$ \\
Diffraction Limit & $1 \mu \mathrm{m}$ & $1 \mu \mathrm{m}$ & $100 \mathrm{fs}$ & $58 \mathrm{EW}$ & $1.8 \times 10^{31} \mathrm{~W} / \mathrm{m}^{2}$ & $8.3 \times 10^{16} \mathrm{~V} / \mathrm{m}$ \\
\hline
\end{tabular}

Table 2: This table displays the minimally required power $P_{\min }$, the corresponding power density $S_{\text {min }}$ and electric field $\mathcal{E}_{\text {min }}$, to produce at least one $e^{+} e^{-}$pair in a volume $\triangle V=\sigma^{3}$ during a time interval $\triangle t$ at the focus of a laser with spot radius $\sigma\left(1 \mathrm{EW}=10^{18} \mathrm{~W}\right)$.

a laser with spot radius $\sigma$ are given in Table 2. This table demonstrates clearly that in order to get a sizeable effect it is mandatory to reach the diffraction limit of focusing at X-ray lasers. We note that research and development in this direction is under way [33, 45].

As discussed at the end of the last section, for $\mathcal{E}>\mathcal{E}_{\min }$ the system is expected to enter rather rapidly the plasma regime. In this sense, the power densities $S_{\min }$ in Table 2 represent also theoretical upper limits for laser fields.

5. We conclude that the power densities and electric fields which can be reached with presently available technique (column labeled "Focus: Available" in Table 1) are far too small to lead to a sizeable effect. On the other hand, if X-ray optics can be considerably improved, allowing the theoretical diffraction limit to be reached, and if the FEL power can be increased from the presently planned values to the terawatt regime, as has been argued to be possible by the tapered undulator technique [37, then there is ample room (c.f. column labeled "Focus: Goal" in Table 1) for an investigation of the Schwinger pair production mechanism at a future X-ray FEL. Intensive development in technical areas, particularly in that of X-ray optics, will be needed in order to achieve the required ultrahigh power densities [33, 45]. It should be pointed out, however, that even though progress to achieve such a lofty goal is rather slow and laborious, the rewards that may be derived in this unique regime are so extraordinary that looking into XFEL's or LCLS's extension to this regime merits serious considerations. There will be unprecedented opportunities to use these intense X-rays in order to explore some issues of fundamental physics that have eluded man's probing so far [39].

\section{Acknowledgements}

I wish to thank P. Zerwas for drawing my attention to this subject. Furthermore, many discussions with, and fruitful comments from, my colleagues at HASYLAB, W. Graeff and G. Materlik, are acknowledged. Moreover, I thank P. Chen, C. Pellegrini and T. Tajima for encouragement, and J. Baacke, W. Buchmüller, M. G. Schmidt and F. Schrempp for a careful reading of the manuscript.

\section{References}


[1] F. Sauter, Z. Phys. 69 (1931) 742.

[2] W. Heisenberg and H. Euler, Z. Phys. 98 (1936) 714.

[3] J. Schwinger, Phys. Rev. 82 (1951) 664.

[4] S. W. Hawking, Commun. Math. Phys. 43 (1975) 199.

[5] T. Damour and R. Ruffini, Phys. Rev. D 14 (1976) 332.

[6] G. W. Gibbons and M. J. Perry, Proc. Roy. Soc. Lond. A 358 (1978) 467.

[7] S. P. Gavrilov and D. M. Gitman, Phys. Rev. D 53 (1996) 7162.

[8] A. Casher, H. Neuberger and S. Nussinov, Phys. Rev. D 20 (1979) 179.

[9] B. Andersson, G. Gustafson, G. Ingelman and T. Sjöstrand, Phys. Rept. 97 (1983) 31.

[10] T. S. Biro, H. B. Nielsen and J. Knoll, Nucl. Phys. B 245 (1984) 449.

[11] L. Parker, Phys. Rev. 183 (1969) 1057.

[12] N. D. Birrell and P. C. Davies, Quantum Fields in Curved Space (Cambridge University Press, 1982).

[13] W. Greiner, B. Müller and J. Rafelski, Quantum Electrodynamics of Strong Fields (SpringerVerlag, Berlin, 1985).

[14] A. A. Grib, S. G. Mamaev and V. M. Mostepanenko, Vacuum Quantum Effects in Strong Fields (Atomizdat, Moscow, 1988; Friedmann Laboratory Publishing, St. Petersburg, 1994).

[15] E. S. Fradkin, D. M. Gitman and Sh. M. Shvartsman, Quantum Electrodynamics with Unstable Vacuum (Springer-Verlag, Berlin, 1991).

[16] Ya. B. Zel'dovich and V. S. Popov, Usp. Fiz. Nauk 105 (1971) 403 [Sov. Phys. Usp. 14 (1972) $673]$.

[17] B. Müller, J. Rafelski and W. Greiner, Z. Phys. 257 (1972) 62 and 183.

[18] W. Greiner and J. Reinhardt, in Quantum Aspects of Beam Physics, Proc. 15th Advanced ICFA Beam Dynamics Workshop, Monterey, Calif., 4-9 Jan 1998, ed. P. Chen (World Scientific, Singapore, 1998), p. 438.

[19] F. V. Bunkin and I. I. Tugov, Dokl. Akad. Nauk Ser. Fiz. 187 (1969) 541 [Sov. Phys. Dokl. 14 (1970) 678].

[20] E. Brezin and C. Itzykson, Phys. Rev. D 2 (1970) 1191.

[21] V. S. Popov, Pisma Zh. Eksp. Teor. Fiz. 13 (1971) 261 [JETP Lett. 13 (1971) 185].

[22] V. S. Popov, Zh. Eksp. Teor. Fiz. 61 (1971) 1334 [Sov. Phys. JETP 34 (1972) 709]. 
[23] G. J. Troup and H. S. Perlman, Phys. Rev. D 6 (1972) 2299.

[24] V. S. Popov, Zh. Eksp. Teor. Fiz. 62 (1972) 1248 [Sov. Phys. JETP 35 (1972) 659].

[25] V. S. Popov and M. S. Marinov, Yad. Fiz. 16 (1972) 809 [Sov. J. Nucl. Phys. 16 (1973) 449].

[26] N. B. Narozhnyi and A. I. Nikishov, Zh. Eksp. Teor. Fiz. 65 (1973) 862 [Sov. Phys. JETP 38 (1974) 427].

[27] V. S. Popov, Pisma Zh. Eksp. Teor. Fiz. 18 (1973) 435 [JETP Lett. 18 (1974) 255].

[28] V. M. Mostepanenko and V. M. Frolov, Yad. Fiz. 19 (1974) 885 [Sov. J. Nucl. Phys. 19 (1974) 451].

[29] V. S. Popov, Yad. Fiz. 19 (1974) 1140 [Sov. J. Nucl. Phys. 19 (1974) 584].

[30] M. S. Marinov and V. S. Popov, Fortsch. Phys. 25 (1977) 373.

[31] R. Brinkmann, G. Materlik, J. Rossbach and A. Wagner, Hamburg, Germany: DESY (1997) 1183 p. Hamburg DESY - DESY-97-048 (97/05,rec.Sep.) 1183 p. (ECFA 97-182).

[32] G. Materlik and T. Wroblewski, in Workshop On The Development Of Future Linear Electron-Positron Colliders For Particle Physics Studies And For Research Using Free Electron Lasers, eds. G. Jarlskog, U. Mjörnmark and T. Sjöstrand (Lund University, 1999), pp. 39-58.

[33] TESLA - The Superconductiong Electron Positron Linear Collider with an Integrated XRay Laser Laboratory, Technical Design Report, DESY 2001-011, ECFA 2001-209, TESLAReport 2001-23, TESLA-FEL 2001-05.

[34] J. Arthur et al. [LCLS Design Study Group Collaboration], SLAC-R-0521 (1998).

[35] I. Lindau, M. Cornacchia and J. Arthur, in Workshop On The Development Of Future Linear Electron-Positron Colliders For Particle Physics Studies And For Research Using Free Electron Lasers, eds. G. Jarlskog, U. Mjörnmark and T. Sjöstrand (Lund University, 1999), pp. 153-161.

[36] A. C. Melissinos, in Quantum Aspects of Beam Physics, Proc. 15th Advanced ICFA Beam Dynamics Workshop, Monterey, Calif., 4-9 Jan 1998, ed. P. Chen (World Scientific, Singapore, 1998), p. 564.

[37] P. Chen and C. Pellegrini, in Quantum Aspects of Beam Physics, Proc. 15th Advanced ICFA Beam Dynamics Workshop, Monterey, Calif., 4-9 Jan 1998, ed. P. Chen (World Scientific, Singapore, 1998), p. 571.

[38] P. Chen and T. Tajima, Phys. Rev. Lett. 83 (1999) 256.

[39] T. Tajima, "Fundamental Physics with an X-Ray Free Electron Laser," Comments on Plasma Physics and Controlled Fusion (2001), to appear. 
[40] D. L. Burke et al., Phys. Rev. Lett. 79 (1997) 1626.

[41] C. Bamber et al., Phys. Rev. D 60 (1999) 092004.

[42] D. E. Groom et al. [Particle Data Group Collaboration], Eur. Phys. J. C 15 (2000) 1.

[43] M. Perry and G. Mourou, Science 264 (1994) 917.

[44] W. Graeff, private communication.

[45] J. B. Hastings et al., "X-ray Laser Physics", in LCLS - The First Experiments (September 2000).

[46] G. Dunne and T. Hall, Phys. Rev. D 58 (1998) 105022.

[47] L. Kofman, A. Linde and A. A. Starobinsky, Phys. Rev. D 56 (1997) 3258.

[48] A. Erdelyi, Higher Transcendental functions, Volume II, by A. Erdelyi (ed.) , W. Magnus (ed.) , F. Oberhettinger (ed.) and F. G. Tricomi (ed.) (McGraw-Hill, 1953).

[49] I. D. Novikov and A. A. Starobinskii, Zh. Eksp. Teor. Fiz. 78 (1980) 3 [Sov. Phys. JETP 51 (1980) 1].

[50] B. L. Spokoinyi, Yad. Fiz. 36 (1982) 474 [Sov. J. Nucl. Phys. 36 (1982) 277].

[51] F. Cooper and E. Mottola, Phys. Rev. D 40 (1989) 456.

[52] Y. Kluger, J. M. Eisenberg, B. Svetitsky, F. Cooper and E. Mottola, Phys. Rev. Lett. 67 (1991) 2427.

[53] Y. Kluger, J. M. Eisenberg, B. Svetitsky, F. Cooper and E. Mottola, Phys. Rev. D 45 (1992) 4659 .

[54] Y. Kluger, J. M. Eisenberg and B. Svetitsky, Int. J. Mod. Phys. E2 (1993) 333.

[55] Y. Kluger, E. Mottola and J. M. Eisenberg, Phys. Rev. D 58 (1998) 125015.

[56] S. M. Schmidt, D. Blaschke, G. Röpke, S. A. Smolyansky, A. V. Prozorkevich and V. D. Toneev, Int. J. Mod. Phys. E7 (1998) 709.

[57] S. M. Schmidt, D. Blaschke, G. Röpke, A. V. Prozorkevich, S. A. Smolyansky and V. D. Toneev, Phys. Rev. D 59 (1999) 094005. 